\title{
The effect of in situ/in vitro three- dimensional quantitative computed tomography image voxel size on the finite element model of human vertebral cancellous bone
}

Proc IMechE Part H:

$\mathrm{J}$ Engineering in Medicine

2014, Vol. 228(II) 1208-1213

(C) IMechE 2014

Reprints and permissions:

sagepub.co.uk/journalsPermissions.nav DOI: $10.1177 / 0954411914558654$

pih.sagepub.com

(S)AGE

\author{
Yongtao Lu',2, Klaus Engelke ${ }^{3}$, Claus-C Glueer ${ }^{4}$, Michael M Morlock' and \\ Gerd Huber'
}

\begin{abstract}
Quantitative computed tomography-based finite element modeling technique is a promising clinical tool for the prediction of bone strength. However, quantitative computed tomography-based finite element models were created from image datasets with different image voxel sizes. The aim of this study was to investigate whether there is an influence of image voxel size on the finite element models. In all 12 thoracolumbar vertebrae were scanned prior to autopsy (in situ) using two different quantitative computed tomography scan protocols, which resulted in image datasets with two different voxel sizes $\left(0.29 \times 0.29 \times 1.3 \mathrm{~mm}^{3}\right.$ vs $\left.0.18 \times 0.18 \times 0.6 \mathrm{~mm}^{3}\right)$. Eight of them were scanned after autopsy (in vitro) and the datasets were reconstructed with two voxel sizes $\left(0.32 \times 0.32 \times 0.6 \mathrm{~mm}^{3}\right.$ vs. $\left.0.18 \times 0.18 \times 0.3 \mathrm{~mm}^{3}\right)$. Finite element models with cuboid volume of interest extracted from the vertebral cancellous part were created and inhomogeneous bilinear bone properties were defined. Axial compression was simulated. No effect of voxel size was detected on the apparent bone mineral density for both the in situ and in vitro cases. However, the apparent modulus and yield strength showed significant differences in the two voxel size group pairs (in situ and in vitro). In conclusion, the image voxel size may have to be considered when the finite element voxel modeling technique is used in clinical applications.
\end{abstract}

\author{
Keywords \\ Quantitative computed tomography image, finite element, voxel size, in situ, in vitro, vertebra
}

Date received: II August 2014; accepted: 15 October 2014

\section{Introduction}

In recent years, finite element (FE) modeling technique based on three-dimensional (3D) quantitative computed tomography (QCT) data have showed great potentials for clinical applications in the prediction of bone strength. ${ }^{1-4}$ Compared to the traditional dualenergy X-ray absorptiometry (DXA) technique, the FE modeling approach has showed better predictions for bone fracture. ${ }^{5,6}$ This is because that the FE models have incorporated not only the 3D geometry of bone but also the inhomogeneous distribution of the material properties, which is implemented by mapping the CT value-derived mechanical properties to the FE models. ${ }^{7}$ Different clinical CT scan protocols are currently available for scanning human vertebrae, and consequently QCT-based FE models are generated from image datasets with different voxel sizes. ${ }^{1,8,9}$ However, as a result of the material mapping procedure, the quality of such FE models might be influenced by the image voxel size, which is crucial for defining the anatomic structures. Smaller voxels allow for a more detailed representation

\footnotetext{
'Institute of Biomechanics, Hamburg University of Technology (TUHH), Hamburg, Germany

${ }^{2}$ INSIGNEO Institute for in silico Medicine, Department of Mechanical Engineering, The University of Sheffield, Sheffield, UK

${ }^{3}$ Institute of Medical Physics, University of Erlangen-Nuremberg, Erlangen, Germany

${ }^{4}$ Section Biomedical Imaging, Department of Radiology and

Neuroradiology, Christian-Albrechts Universität zu Kiel, Kiel, Germany
}

\section{Corresponding author:}

Yongtao Lu, INSIGNEO Institute for in silico Medicine, Department of Mechanical Engineering, The University of Sheffield, Mappin Street, Sheffield SI 3JD, UK.

Email: yongtao.lu@sheffield.ac.uk; bennett200I@gmail.com 
of the geometry and material properties, but require high radiation doses exposed to the patient. ${ }^{10}$ On the other hand, larger voxels require lower radiation doses, but cannot resolve smaller structures like single trabecular at clinical QCT resolutions and consequently could cause partial volume artifacts. ${ }^{10}$ When the FE modeling technique is used in clinical applications, the image voxel size and patient radiation dose need to be balanced. However, if the influence of image voxel size on the FE models is small and negligible, CT datasets with larger voxel size can be considered to reduce the patient radiation dose.

The aim of this study was to investigate the influence of in situ/in vitro 3D QCT image voxel size on the FE models of human vertebral cancellous bone.

\section{Materials and methods}

\section{In situ/in vitro QCT scans}

Female cadavers were harvested from 35 female patients with a mean age of $81.3 \pm 7.2$ years (range: $65-$ 90 years). Within $7.2 \pm 3.1$ days after death and prior to autopsy, the cadavers were scanned on a clinical whole body CT scanner (MX8000; Philips, Best, The Netherlands) referred as in situ scan in this article. The in situ scans were performed using two protocols: a standard protocol- $120 \mathrm{kV}, 100 \mathrm{mAs}$, field of view of $15 \mathrm{~cm}$, slice thickness of $1.3 \mathrm{~mm}$, scan range including vertebrae from T6 to L4; and a high-resolution protocol- $120 \mathrm{kV}, 360 \mathrm{~mA} \mathrm{~s}$, field of view of $9 \mathrm{~cm}$, slice thickness of $0.6 \mathrm{~mm}$, scan range only including $\mathrm{T} 12$ with adjacent endplates of T11 and L1. The cadavers were not repositioned in between the two scanning procedures. The datasets from the standard protocol were reconstructed with a voxel size of $0.29 \times 0.29 \times 1.3$ $\mathrm{mm}^{3}$ and those from the high-resolution protocol were reconstructed with a voxel size of $0.18 \times 0.18 \times 0.6$ $\mathrm{mm}^{3}$. A solid calibration phantom (QRM Bone Density Calibration (BDC) phantom; QRM GmbH, Möhrendorf, Germany), which has the density in terms of hydroxyapatite (CaHA), was placed beneath the cadavers.

After the in situ scan, the spinal segment from T10 to L1 was retrieved from each cadaver. Each segment was wrapped into a towel moistened with physiological salt solution, double-sealed in a plastic bag and kept frozen below $-20^{\circ} \mathrm{C}$. On the day of in vitro scanning, the specimens were surrounded by tissue-equivalent bolus bags and scanned on a clinical CT scanner (Sensation 64; Siemens, Erlangen, Germany) using $120 \mathrm{kV}, 360 \mathrm{mAs}$ and slice thickness of $0.6 \mathrm{~mm}$, referred as in vitro scan in this article. The datasets were reconstructed with voxel sizes of $0.32 \times 0.32 \times 0.6 \mathrm{~mm}^{3}$ and $0.18 \times 0.18 \times 0.3 \mathrm{~mm}^{3}$. A calibration phantom (Mindways, San Francisco, CA, USA), which has the density in terms of dipotassium hydrogen phosphate $\left(\mathrm{K}_{2} \mathrm{HPO}_{4}\right)$, was placed beneath the specimens.
The thoracolumbar vertebra T12 was chosen for investigation. Specimens with present T12 fractures or pathologies were excluded. Out of the given vertebrae, 12 QCT datasets were remained for the in situ analysis, 8 of them remained for the in vitro analysis due to the faults (damage during autopsy, non-consistent scan protocol, etc.) in some specimens.

\section{Density calibration between phantoms}

As the relationship between the CaHA density and bone mechanical properties was used in this article, the $\mathrm{K}_{2} \mathrm{HPO}_{4}$ density had to be converted to the CaHA density. To do this, the QRM BDC phantom was placed on top of the Mindways phantom and the two phantoms were repeatedly scanned six times on the Sensation 64 CT scanner using the in vitro scan protocol. The calculated $\mathrm{CaHA}$ and $\mathrm{K}_{2} \mathrm{HPO}_{4}$ density values were linearly correlated, and the regression intercept of the CaHA and $\mathrm{K}_{2} \mathrm{HPO}_{4}$ densities was assumed to be $0 \mathrm{mgCaHA} / \mathrm{cm}^{3}$. It is believed that the use of two calibration phantoms should not influence the conclusions made in this article, because no comparisons between in situ and in vitro models were made in this article.

\section{FE voxel model}

Following the published procedures, ${ }^{11}$ the cuboid volume of interest (VOI) with the size of approximately $12.3 \mathrm{~mm} \times 12.3 \mathrm{~mm} \times 14.3 \mathrm{~mm}$, which was chosen to cover the largest possible trabecular region within a vertebral body, was cropped from the center of the vertebral bodies (Avizo V5.0; Mercury Computer Systems, Inc., Burlington, MA, USA). The FE voxel model was created by converting each image voxel into a hexahedral element (C3D8) (Figure 1) and then was refined until convergent results were achieved (Figure 2). Inhomogeneous, bilinear material properties were assigned to the FE model. ${ }^{12}$ The behavior of the cuboid VOI was investigated under the uniaxial compression scenario, and the apparent modulus and yield strength were output from the FE analysis (Abaqus V6.11; Dassault Systems SIMULIA Ltd, Providence, RI, USA). ${ }^{11}$

\section{Statistical analysis}

All the data were checked for normality using the Shapiro-Wilk test. The datasets were grouped into two pairs: in situ and in vitro. A paired sample t-test was used to compare the bone mineral density (BMD), the FE calculated apparent modulus and yield strength within each pair. The significance level was set to $95 \%$ $(\alpha=0.05)$. A Bonferroni correction was used to account for multiple comparisons. The Bonferroni-corrected p-value was $0.05 / 2=0.025$. Regression equations and coefficients of determination $\left(\mathrm{R}^{2}\right)$ were computed for the linear correlations of the apparent 


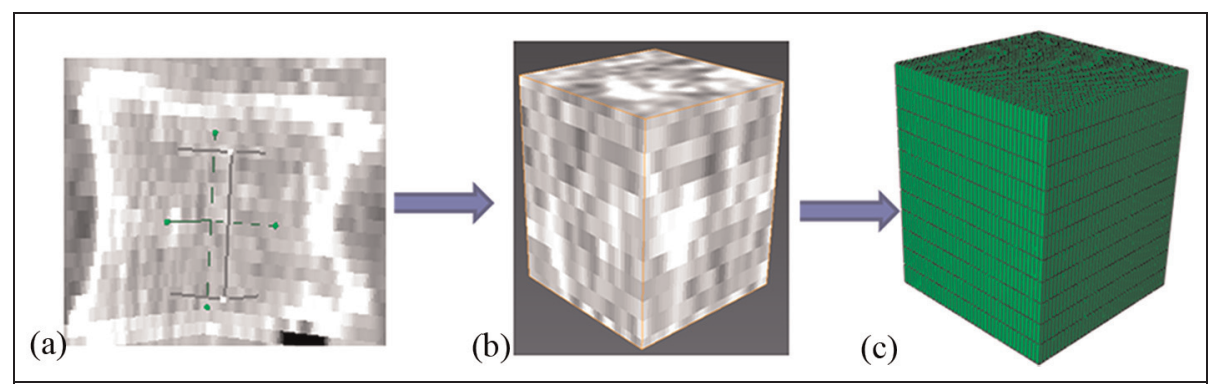

Figure I. Generation of the finite element (FE) voxel model from the QCT dataset: (a) vertebral body with cuboid volume of interest (VOI) (solid gray lines), (b) grayscale images of VOI extracted from the center of vertebral body and (c) corresponding FE voxel model.

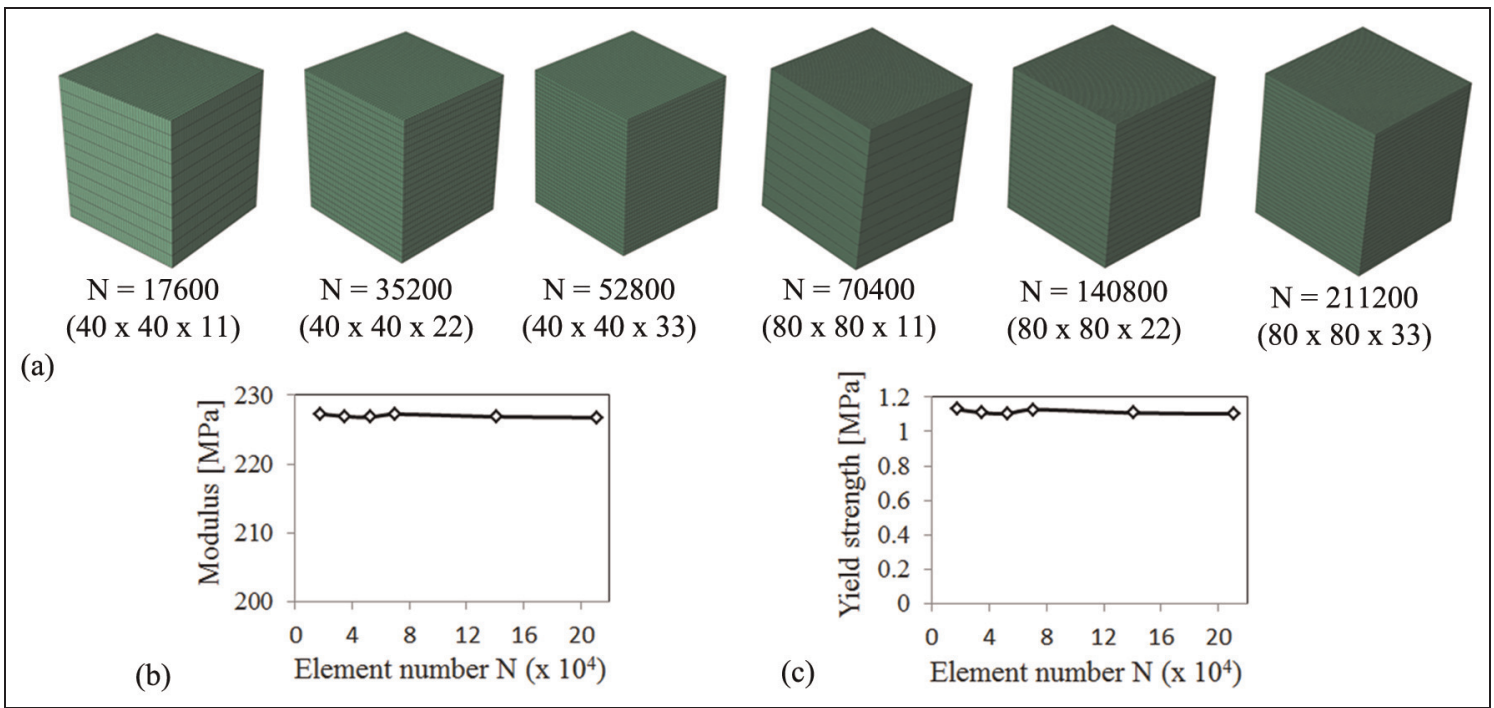

Figure 2. A representative mesh convergence study (the in situ low-resolution case): (a) illustration of the meshes from coarsest (one-to-one mapping between image voxel and finite element) to finest, (b) convergence of the modulus and (c) convergence of the yield strength of the cuboid volume of interest.

modulus and yield strength with the BMD. Quality of the fit to the line $\mathrm{y}=\mathrm{x}$ was given by the concordance correlation coefficient (CCC). ${ }^{13}$

\section{Results}

The mean value \pm standard deviation (SD) for the linear regression slope between the two kinds of phantoms was $0.91 \pm 0.01$, that is, $\operatorname{BMD}(\mathrm{CaHA})=0.91$ $\mathrm{BMD}\left(\mathrm{K}_{2} \mathrm{HPO}_{4}\right)$.

No effect of image voxel size on the apparent BMD was detected for the in situ pair $(\mathrm{p}=0.126)$ and for the in vitro pair $(\mathrm{p}=0.114)$ (Figure 3$)$. However, the voxel size had a significant effect on the apparent modulus and yield strength. Comparing values in the high- and low-resolution pair, for the in situ case, the apparent modulus was $197.17 \pm 43.32 \mathrm{MPa}$ versus $209.67 \pm$ $50.80 \mathrm{MPa}(\mathrm{p}=0.001)$ and the yield strength was $1.01 \pm 0.27 \mathrm{MPa}$ versus $1.07 \pm 0.34 \mathrm{MPa} \quad(\mathrm{p}=0.009)$, and for the in vitro case, the apparent modulus was $136.63 \pm 46.63 \mathrm{MPa}$ versus $149.87 \pm 45.49 \mathrm{MPa}$ $(\mathrm{p}<0.001)$ and the yield strength was $0.64 \pm 0.28 \mathrm{MPa}$ versus $0.74 \pm 0.28 \mathrm{MPa}(\mathrm{p}<0.001)$. Apparent modulus and yield strength were linearly correlated in the two resolution groups both for the in situ and in vitro cases (Figures 4 and 5). Moderate agreement between the two resolution groups was obtained $(\mathrm{CCC}=0.93-$ 0.95), and the Bland-Altman plots showed there were consistent biases and both the modulus and the yield strength increased with the increasing voxel size (Figures 4 and 5).

\section{Discussion}

In this study, first it is shown that the QCT image voxel size had no effect on the apparent BMD. In this study, within each in situ/in vitro comparison pair, the datasets were acquired with the same tube voltage, which is a factor influencing the CT values and consequently the BMD. ${ }^{14}$ Although in the in situ pair, the datasets were acquired at different tube currents, a change in tube current at a given tube voltage changes the image noise, 


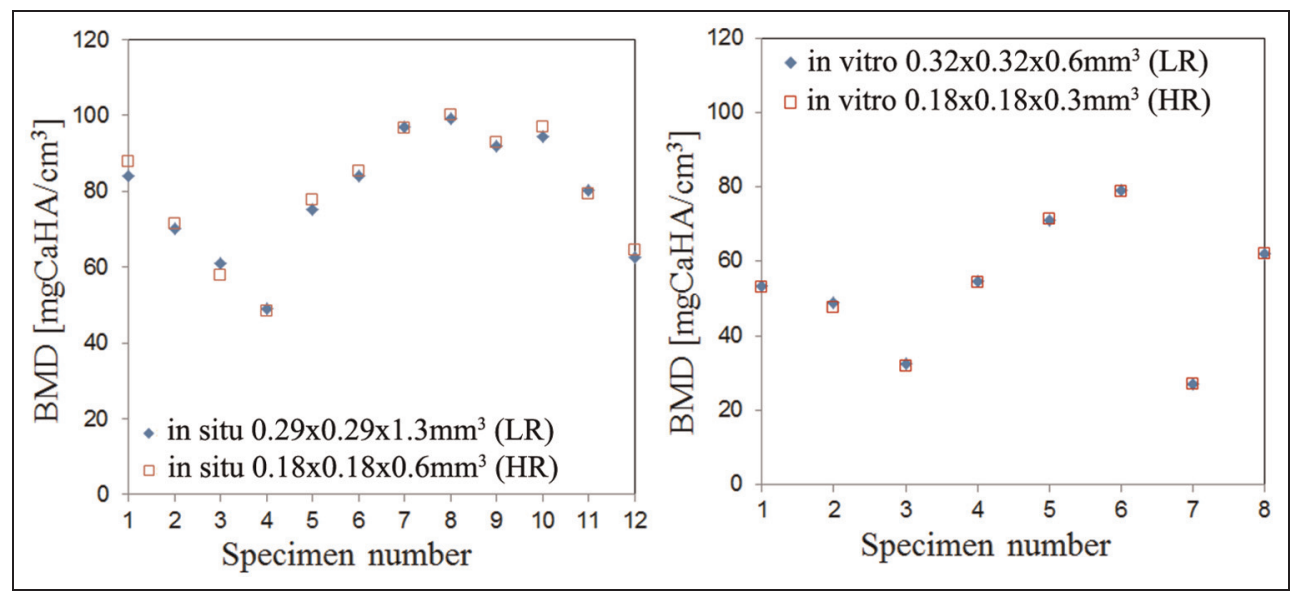

Figure 3. Comparisons of the apparent BMDs averaged over the cuboid volume of interest in the low-resolution (LR) and highresolution (HR) measurement groups for the in situ (left) and in vitro (right) cases.

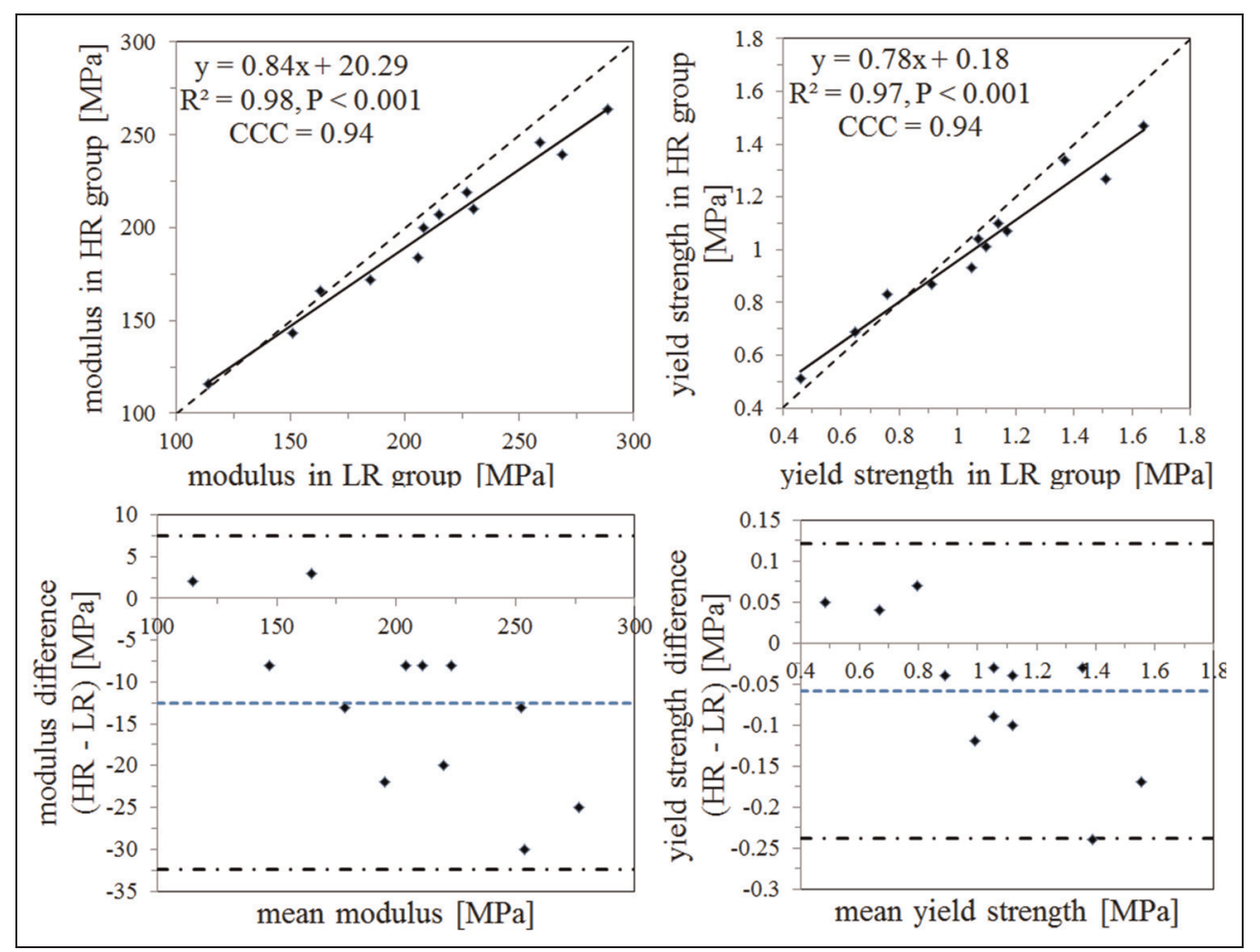

Figure 4. Linear regression (solid line), concordance correlation coefficients (CCC) and Bland-Altman diagrams (average difference \pm 1.96 standard deviation of the difference) of modulus and yield strength in high-resolution $\left(H R ; 0.18 \times 0.18 \times 0.6 \mathrm{~mm}^{3}\right)$ and low-resolution $\left(\mathrm{LR} ; 0.29 \times 0.29 \times 1.3 \mathrm{~mm}^{3}\right)$ groups for the in situ case.

but not the mean BMD value obtained, for example, in the trabecular compartment of a vertebra. ${ }^{15}$ Not surprisingly, the difference in BMD within each pair was not significant. Second, this study showed that there is a significant effect of the QCT image voxel size on FEcalculated apparent modulus and yield strength, which is in agreement with previous publications. ${ }^{16-18}$ However, these previous studies did not resemble the clinical scenario, due to the facts that the micro-FE models were investigated and some low-resolution image datasets were obtained by numerically down- sampling the high-resolution images. ${ }^{17,18}$ Consequently, their conclusions cannot be of direct clinical relevance. To the authors' knowledge, the study performed in this article was the first to investigate the influence of QCT voxel size on the FE models at the clinical resolution level, although only high-resolution QCT images were investigated. Nevertheless, an impact of voxel size on the FE predictions was found. It should be noted that if the image resolution in the low-resolution group was as low as the cuboid size, then the model becomes homogeneous isotropic and the comparisons become between 


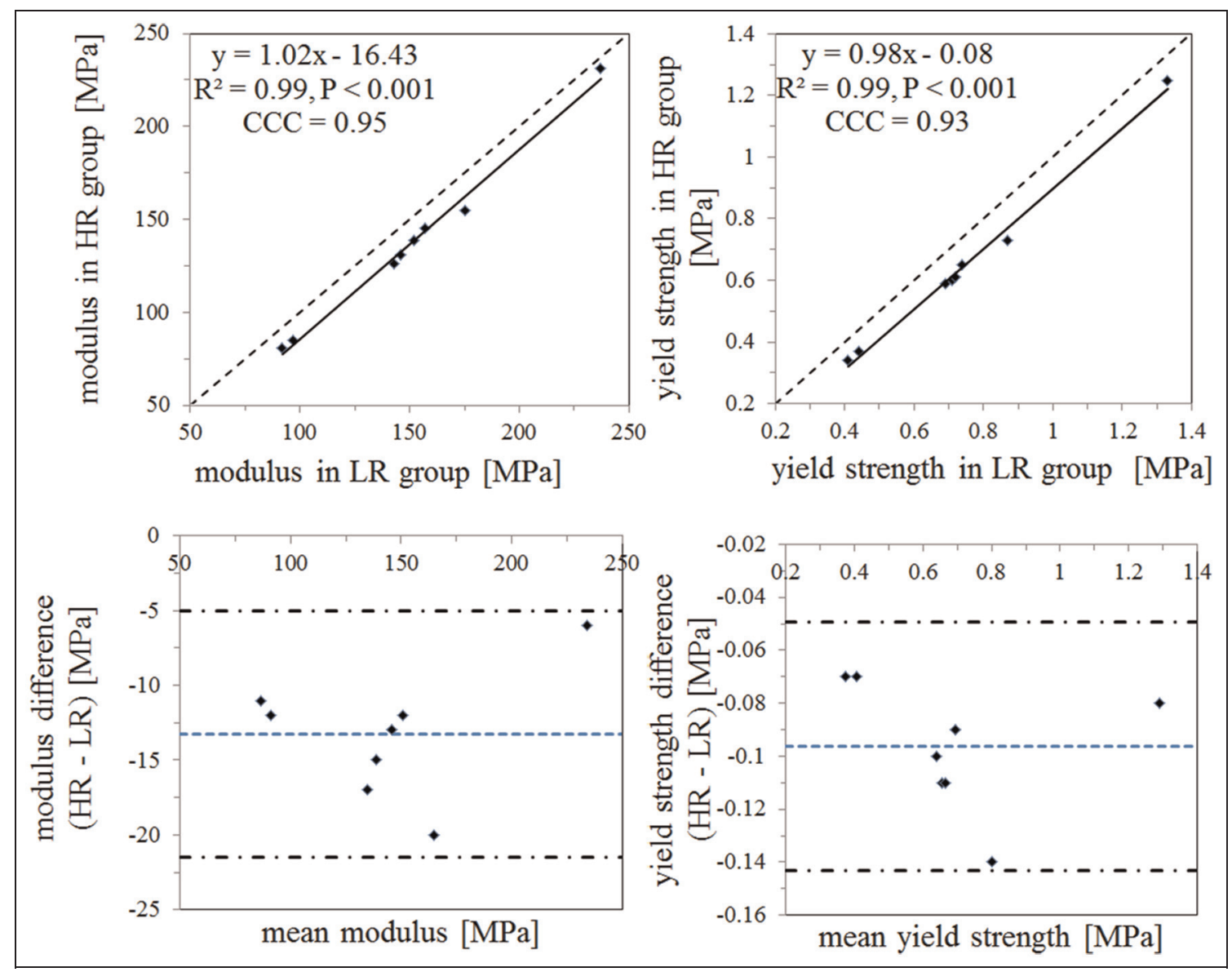

Figure 5. Linear regression (solid line), concordance correlation coefficients (CCC) and Bland-Altman diagrams of modulus and yield strength in high-resolution $\left(\mathrm{HR} ; 0.18 \times 0.18 \times 0.3 \mathrm{~mm}^{3}\right)$ and low-resolution $\left(\mathrm{LR} ; 0.32 \times 0.32 \times 0.6 \mathrm{~mm}^{3}\right)$ groups for the in vitro case.

the homogeneous and heterogeneous isotropic FE models. In this sense, the conclusion that increased image voxel size leads to overestimation of elastic properties is in agreement with the published studies. ${ }^{19-24} \mathrm{It}$ should also be noted that for the in situ groups, different voxel sizes were resulted from both different image acquisition and different reconstruction parameters, while for the in vitro groups, they only resulted from different reconstruction parameters.

A few limitations in this article should be discussed. First, a specific CT-to-elasticity relationship, ${ }^{12}$ which was obtained from the vertebral body samples, was used. It should be noted that the scan protocol in Kopperdahl et al. ${ }^{12}$ is different as the ones used in this article, which may cause some errors in the FE-derived values. ${ }^{11}$ However, these errors were added to both the high- and low-resolution groups, which could make their effect negligible. Second, with the applied CT-toelasticity relationship, negative Young's moduli are reached for small $\mathrm{BMD}$ values. To avoid the occurrence of this situation, a threshold of $10 \mathrm{mgCaHA} / \mathrm{cm}^{3}$ was applied to the CT-to-elasticity function. It is worthy to mention that the aforementioned limitations could be solved by applying the micromechanic-based approaches to derive the CT-to-elasticity relations. ${ }^{19,20,25-28}$

In conclusion, this study suggested that the 3D QCT-based FE voxel models created from in situ/in vitro image datasets with different voxel sizes would give significantly different results. This study provided important insights into the clinical applications of FE modeling technique by implying that consistent QCT image voxel size should be used when using the FE models to predict the in vivo bone strength.

\section{Acknowledgements}

The authors would like to acknowledge the Department of Legal Medicine, University Medical Center Hamburg-Eppendorf, Hamburg, Germany, for providing the in situ QCT image datasets; Dr Peter Varga for some technical supports and Miss Imke Fiedler for assisting in the BMD and FE calculations.

\section{Declaration of conflicting interests}

The authors declare that there is no conflict of interest. 


\section{Funding}

This study was supported by the German Federal Ministry of Education and Research (BMBF) through the consortium "BioAsset" (Grant number 01EC1005).

\section{References}

1. Chevalier Y, Quek E, Borah B, et al. Biomechanical effects of teriparatide in women with osteoporosis treated previously with alendronate and risedronate: results from quantitative computed tomography-based finite element analysis of the vertebral body. Bone 2010; 46: 41-48.

2. Mawatari T, Miura H, Hamai S, et al. Vertebral strength changes in rheumatoid arthritis patients treated with alendronate, as assessed by finite element analysis of clinical computed tomography scans: a prospective randomized clinical trial. Arthritis Rheum 2008; 58(1): 33403349.

3. Kleerekoper M, Greenspan SL, Lewiecki EM, et al. Assessing the effects of teriparatide treatment on bone mineral density, bone microarchitecture, and bone strength. J Bone Joint Surg Am 2014; 96(11): e90.

4. Kopperdahl DL, Aspelund T, Hoffmann PF, et al. Assessment of incident spine and hip fractures in women and men using finite element analysis of CT scans. $J$ Bone Miner Res 2014; 29(3): 570-580.

5. Wang X, Sanyal A, Cawthon PM, et al. Prediction of new clinical vertebral fractures in elderly men using finite element analysis of CT scans. J Bone Miner Res 2012; 27(4): 808-816.

6. Imai K, Ohnishi I, Matsumoto T, et al. Assessment of vertebral fracture risk and therapeutic effects of alendronate in postmenopausal women using a quantitative computed tomography-based nonlinear finite element method. Osteoporos Int 2009; 20: 801-810.

7. Chen G, Schmutz B, Epari D, et al. A new approach for assigning bone material properties from $\mathrm{CT}$ images into finite element models. J Biomech 2010; 43: 1011-1015.

8. Crawford RP, Cann CE and Keaveny TM. Finite element models predict in vitro vertebral body compressive strength better than quantitative computed tomography. Bone 2003; 33: 744-750.

9. Pahr DH, Schwiedrzik J, Dall'Ara E, et al. Clinical versus pre-clinical FE models for vertebral body strength predictions. J Mech Behav Biomed Mater 2014; 33: 7683.

10. Suetens P. Fundamentals of medical imaging. 2nd ed.New York: Cambridge University Press, 2009.

11. Lu Y, Engelke K, Puschel K, et al. Influence of 3D QCT scan protocol on the QCT-based finite element models of human vertebral cancellous bone. Med Eng Phys 2014; 36(8): 1069-1073.

12. Kopperdahl DL, Morgan EF and Keaveny TM. Quantitative computed tomography estimates of the mechanical properties of human vertebral trabecular bone. J Orthop Res 2002; 20(4): 801-805.
13. Lin LK. A concordance correlation coefficient to evaluate reproducibility. Biometrics 1989; 45: 255-268.

14. Museyko O, Heinemann A, Krause M, et al. A low radiation exposure protocol for 3D QCT of the spine. Osteoporos Int 2014; 25(3): 983-992.

15. Bui AAT and Taira RK. Medical Imaging Informatics. New York: Springer, 2010.

16. Yeni YN, Christopherson GT, Dong XN, et al. Effect of microcomputed tomography voxel size on the finite element model accuracy for human cancellous bone. $J$ Biomech Eng 2005; 127(1): 1-8.

17. Bevill $\mathrm{G}$ and Keaveny TM. Trabecular bone strength predictions using finite element analysis of micro-scale images at limited spatial resolution. Bone 2009; 44(4): 579-584.

18. Maloul A, Fialkov J and Whyne C. The impact of voxel size-based inaccuracies on the mechanical behavior of thin bone structures. Ann Biomed Eng 2011; 39(3): 1092 1100.

19. Dejaco A, Komlev VS, Jaroszewicz J, et al. Micro CTbased multiscale elasticity of double-porous (pre-cracked) hydroxyapatite granules for regenerative medicine. $J$ Biomech 2012; 45: 1068-1075.

20. Blanchard R, Dejaco A, Bongaers E, et al. Intravoxel bone micromechanics for microCT-based finite element simulations. J Biomech 2013; 46: 2710-2721.

21. Baca V, Horak Z, Mikulenka P, et al. Comparison of an inhomogeneous orthotropic and isotropic material models used for FE analyses. Med Eng Phys 2008; 30(7): 924 930.

22. Wroe S, Moreno K, Clausen P, et al. High-resolution three-dimensional computer simulation of hominid cranial mechanics. Anat Rec 2007; 290(10): 1248-1255.

23. Schneider R, Faust G, Hindenlang U, et al. Inhomogeneous, orthotropic material model for the cortical structure of long bones modelled on the basis of clinical CT or density data. Comput Methods Appl Mech Eng 2009; 198: 2167-2174.

24. Renders GAP, Mulder L, Langenbach GEJ, et al. Biomechanical effect of mineral heterogeneity in trabecular bone. J Biomech 2008; 41: 2793-2798.

25. Scheiner S, Sinibaldi R, Pichler B, et al. Micromechanics of bone tissue-engineering scaffolds, based on resolution error-cleared computer tomography. Biomaterials 2009; 30: 2411-2419.

26. Vuong $\mathbf{J}$ and Hellmich $\mathrm{C}$. Bone fibrillogenesis and mineralization: quantitative analysis and implications for tissue elasticity. $J$ Theor Biol 2011; 287: 115-130.

27. Hellmich C, Kober C and Erdmann B. Micromechanicsbased conversion of CT data into anisotropic elasticity tensors, applied to FE simulations of a mandible. Ann Biomed Eng 2008; 36(1): 108-122.

28. Yosibash Z, Trabelsi N and Hellmich C. Subject-specific p-FE analysis of the proximal femur utilizing micromechanics-based material properties. Int $J$ Multiscale Com 2008; 6(5): 483-498. 